\title{
Intranet-Based Quality Improvement Documentation at the Veterans Affairs Maryland Health Care System
}

Andrew Borkowski, M.D., Dong H. Lee, M.D., Debra L. Sydnor, C.T. (A.S.C.P.) I.A.C.,

Robert J. Johnson, M.D., Ph.D., Albert Rabinovitch, M.D., Ph.D., G. William Moore, M.D., Ph.D.

Pathology and Laboratory Medicine Service (AB, DHL, DLS, RJJ, GWM), Veterans Affairs Maryland Health Care System, Baltimore, Maryland; Department of Pathology (AB, RJJ, GWM), University of Maryland

School of Medicine, Baltimore, Maryland; Laboratory Accreditation Program (AR), College of American

Pathologists, Northfield, Illinois; and Department of Pathology (GWM), The Johns Hopkins Medical

Institutions, Baltimore, Maryland

The Pathology and Laboratory Medicine Service of the Veterans Affairs Maryland Health Care System is inspected biannually by the College of American Pathologists (CAP). As of the year 2000, all documentation in the Anatomic Pathology Section is available to all staff through the VA Intranet. Signed, supporting paper documents are on file in the office of the department chair. For the year 2000 CAP inspection, inspectors conducted their document review by use of these Web-based documents, in which each CAP question had a hyperlink to the corresponding section of the procedure manual. Thus inspectors were able to locate the documents relevant to each question quickly and efficiently. The procedure manuals consist of 87 procedures for surgical pathology, 52 procedures for cytopathology, and 25 procedures for autopsy pathology. Each CAP question requiring documentation had from one to three hyperlinks to the corresponding section of the procedure manual. Intranet documentation allows for easier sharing among decentralized institutions and for centralized updates of the laboratory documentation. These documents can be upgraded to allow for multimedia presentations, including text search for key words, hyperlinks to other documents, and images, audio, and video. Use of Web-based documents can improve the efficiency of the inspection process.

Copyright () 2001 by The United States and Canadian Academy of Pathology, Inc.

VOL. 14, NO. 1, P. 1, 2001 Printed in the U.S.A

Date of acceptance: August 23, 2000.

Address reprint requests to: G. William Moore, M.D., Ph.D., Quality Improvement Section, Anatomic Pathology, Pathology and Lab Medicine Service (113), Veterans Affairs Medical Center, 10 North Greene Street, Baltimore, MD 21201-1524; fax: 1-410-433-6324.
KEY WORDS: Internet, Laboratory accreditation, Quality improvement.

Mod Pathol 2001;14(1):1-5

The most recent edition of a major textbook of surgical pathology has observed that the two major changes in pathology practice over the past decade have been increased numbers of special stains, particularly immunostains, and a greater extent of regulation of anatomic pathology laboratories by outside agencies $(1,2)$. The Pathology and Laboratory Medicine Service of the Veterans Affairs Maryland Health Care System (VAMHCS) is a geographically decentralized facility that serves veteran patients in Maryland at three hospitals, one rehabilitation center, and four primary-care medical centers. The VAMHCS pathology laboratories are inspected biannually by the College of American Pathologists (CAP) (3). The institution as a whole is inspected triennially by the Joint Commission for the Accreditation of Healthcare Organizations (JCAHO) (4).

The CAP is one of several agencies that perform inspections of anatomic pathology laboratories. One of the conditions of CAP accreditation is that each hospital inspected by the CAP must provide a team of inspectors for another institution of comparable size every 2 years; the inspectors and inspectees receive an itemized checklist of questions in advance, now available on electronic media, and on the Internet $(3,5,6)$. In this manner, the inspectors can prepare for the documents they wish to examine. The inspectees can make certain in advance that the required documents, many of which are stored in various locations, are all available for examination at a central location during the inspection period.

CAP checklist questions are increasing in number and complexity. The current total number of questions for the regular Laboratory Accreditation Program (excluding reproductive, athletic drug, and 
forensic urine drug programs) is 3046. These are not all unique items; identical or very similar items appear in multiple, section-specific checklists (chemistry, hematology, etc.); and the limited service laboratory and blood gas laboratory checklists are derived entirely from other checklists. Recognizing the importance of cytopathology, and to ensure that sufficient attention is devoted to this area during a CAP inspection, this discipline was split from the rest of anatomic pathology into a new checklist in 1995. The anatomic pathology checklist (surgical pathology, histology laboratory, autopsy pathology, and electron microscopy) currently contains 180 questions, whereas the cytopathology checklist has 93 items.

The number of inspectors, as well as the range of skills required to examine the facilities and to document them fairly and completely, has correspondingly increased. At the VAMHCS CAP inspection in 1980, there were five inspectors. In 1990, there were seven inspectors. In 2000, there were 12 inspectors. This is an increasing burden on the system of voluntary inspectors because the inspectors must take time out of their practices to inspect other laboratories. Although some of this increase relates to the additional work of inspection, there is also an active effort to include trainee inspectors to enlarge the pool of experienced inspectors. Although it can be difficult to predict the ideal number of inspectors needed for a particular laboratory, it is preferable to err on the side of too many people to ensure that the inspection is thorough and not rushed. Nevertheless, it is important to attempt to maximize the efficiency of the inspection process, in particular the ability to promptly provide documentation requested by the inspectors.

We designed an internal Web site to make the inspection more efficient. For each question on the CAP checklist (5), the Web site provides a short explanation, followed by a hyperlink (if applicable) that goes directly to the pertinent section of each procedure description in the Anatomic Pathology Section (APS) procedure manual. The signed paper documents were assembled on a table across from the computer monitors, giving the inspectors the option to browse electronically or to review paper copies.

\section{MATERIALS AND METHODS}

The procedure manuals in the Anatomic Pathology Section were originally prepared as paper documents. With computerization of the APS at the Baltimore Veterans Affairs Medical Center in 1989, the previous procedure manual was digitized by use of a scanner and optical character recognition software. Spelling corrections were made on the scanned manual. Updates are now made annually, or as necessary, in compliance with CAP guidelines. Each year, the professional and technical staff members examine the updates, and the staff members sign a signature page in the manual indicating that they have read and understood the manual. Each separate procedure in the paper manual is signed by the departmental administrator, the appropriate section chief, and the departmental chair, and the signed paper document is retained on file in the office of the departmental chair in perpetuity. In this manner, questions regarding previous versions of the manual can be addressed by consulting the filed paper document. The manual is maintained as both a text file, for printing, and as an HTML file, for Intranet access.

The VAMHCS Intranet is an internal Internet resource with an electronic firewall that ensures privacy within the institution. Since 1992, the manual has been posted on the Veterans Information System Technology Architecture (VISTA) computer system throughout the Baltimore campus. Since 1999, the manual has been posted on the VAMHCS Intranet. In preparation for the year 2000 CAP inspection, we obtained the CAP questions on electronic media. The CAP provides the list of questions for each laboratory section undergoing inspection. The questions are available in HTML, Adobe PDF, and Microsoft Word formats at the CAP Web site (3), and are also supplied to participating laboratories as Microsoft Word files on diskette. These files contain the questions, explanatory notes, commentary, and references.

Questions were copied into the hospital computer system as an HTML file appended to the procedure manual. Each CAP question was answered briefly in narrative form, followed by a hyperlink from the question to the appropriate section in the procedure manual. We used Netscape Communicator (at least version 4.5) or Internet Explorer (at least version 5.0). Earlier versions do not accept a procedure manual document as large as ours (nearly 800 kilobytes).

Each procedure contains the essential elements of the National Committee for Clinical Laboratory Standards format $(6,7)$ and includes hyperlinks with the following menu options: forward to the next page; backward to the previous page; return to table of contents; and return to CAP checklist. For example:

QUESTION: 08:1000 PHASE: II

Is the quality improvement program defined and documented for surgical pathology?

RESPONSE: YES, the quality improvement program is defined and documented for surgical pathology.

See PROCEDURE 35: QUALITY IMPROVEMENT IN ANATOMIC PATHOLOGY.

QUALITY IMPROVEMENT IN ANATOMIC PATHOLOGY. (Procedure 35).

NEXT PAGE 
PREVIOUS PAGE

RETURN TO TABLE OF CONTENTS

RETURN TO CAP CHECKLIST

PRINCIPLE OF THE TEST.

Maintenance of high quality of service for optimal patient care.

\section{Specimen Required}

All human tissue excised at surgery, outpatient clinics, and postmortems, fresh or in fixative, along with a filled-out Tissue Examination Form (U. S. Standard Form 515).

\section{Reagents and Instrumentation \\ VISTA computer system.}

\section{Step-by-Step Description}

1. Quarterly Quality Assessment in Anatomic Pathology from the Armed Forces Institute of Pathology...

2. Cytology Proficiency Testing...

3. Peer Review in Surgical Pathology...

4. Invasive Procedures Review Committee (IPRC)..

5. Communications...

6. Timeliness...

7. Daily Surgical Pathology Quality Improvement Conference...

8. Enrollment in pathology review programs...

9. Reviewing prior surgical pathology material...

10. Protocol for handling disparities in diagnosis...

11. Policy for including intradepartmental consultations...

12. Policy for extradepartmental consultations...

If the inspector wished to see the signed paper manual, it was provided on a table across from the computer monitors.

Sample procedures from the VAMHCS Anatomic Pathology Manual are posted on the Johns Hopkins Autopsy Resource (8).

\section{RESULTS}

The procedure manuals in the APS consist of 87 procedures for surgical pathology, 52 procedures for cytopathology, and 25 procedures for autopsy pathology. Each of the CAP questions requiring documentation had from one to three hyperlinks to the corresponding section of the procedure manual. The most frequent hyperlinks were as follows: 21 links to the Cytopathology Quality Improvement Procedure (Procedure 113); 14 links to the VISTA Computer System Procedure (Procedure 122); 11 links to the Surgical Pathology Quality Improvement Procedure (Procedure 35); and 10 links to the
Examination and Description of Specimens Procedure (Procedure 9).

This manual enabled users to quickly view the checklist. There was sufficient time for the inspectors to conduct a more thorough inspection of the laboratory's physical plant, the prepared histology and cytology slides, and the ancillary paper documentation, which was provided near the computer Intranet work stations. The inspection, including the exit summation conference, was concluded by two inspectors in the APS in a single working day. The APS was not found to be deficient, and they were commended by the inspectors for the efficient organization of their documents.

\section{DISCUSSION}

Pathology practice in academic and community hospitals has seen an explosive growth in the amount of regulation of anatomic pathology laboratories by outside agencies over the past decade. The Pathology and Laboratory Medicine Service of the VAMHCS is a geographically decentralized laboratory that is inspected biannually by the CAP; the institution as a whole is inspected triennially by the JCAHO.

The CAP was founded in 1947 and began the Laboratory Accreditation Program in 1962 (9-14). Ten pathology laboratories were inspected in the first cycle. The CAP Commission on Laboratory Accreditation is a component of the CAP Council on Scientific Affairs. The Commissions on Anatomic Pathology, Clinical Pathology, and Quality Assurance also fall under the direction of the CAP Council on Scientific Affairs. The CAP Laboratory Accreditation Program now inspects and accredits more than 6000 hospital, reference, and clinical laboratories throughout the world. Performance monitoring requires the coordination of regional, deputy, and state commissioners in their respective areas with the staff at CAP headquarters in Northfield, IL. State commissioners recruit, train, and assign inspection team leaders to laboratories in their assigned areas.

The CAP maintains a relationship with the U.S. Health Care Financing Administration, an entity of the federal government (15). CAP Laboratory Accreditation Program was granted equivalency when the Clinical Laboratory Improvement Act of 1967 was promulgated as law. The CAP made formal application as a private, nonprofit accrediting agency, with deeming authority under the requirements of the Clinical Laboratory Improvement Amendments of 1988. A relationship was also established with the JCAHO, becoming more formal as the federal requirements changed. JCAHO presently recognizes CAP accreditation as equivalent to its own for laboratories 
within institutions that JCAHO accredits. These relationships require that U.S. Health Care Financing Administration and JCAHO oversee the CAP accreditation process. U.S. Health Care Financing Administration validates CAP inspections with unannounced inspections within 60 days of the CAP on-site visit. JCAHO occasionally sends observers with CAP teams. CAP laboratories are divided into geographic regions, with a commissioner over each. The CAP employs a decentralized structure to foster standards of laboratory accreditation: communication among inspectors; communication among laboratories; problem resolution; and continuing education.

The goal of the CAP Laboratory Accreditation Program is laboratory improvement. It is thought that laboratory improvement will occur through a combination of voluntary peer review, education, and compliance with established standards. Accredited laboratories comply voluntarily. This process identifies excellence by recognizing those laboratories that conform to the four standards of accreditation fostered by the CAP.

Laboratories choosing to voluntarily comply are reviewed by volunteer inspectors acting as peer reviewers. Peer inspectors are paired with laboratories operating under similar size and complexity, with inspection teams composed of pathologists, clinical scientists, medical technologists, cytotechnologists, etc. Both parties participate educationally in a professional exchange of ideas, and both parties benefit. Volunteer inspectors are the Laboratory Accreditation Program's greatest strength as well as one of its greatest challenges. As professionals working in the field, volunteers are able to suggest realistic approaches to laboratory improvement. CAP inspections are conducted by certified, practicing pathologists and technologists who are working in laboratories of a comparable size to the laboratory being inspected. Thus they have firsthand knowledge of the processes in a laboratory. However, it is often difficult for busy professionals to find time to train people and to conduct inspections.

Although this peer approach ensures that evaluations are clinically and scientifically contemporary, it also means that there is greater variation among inspectors than in accreditation programs that use fewer full-time inspectors. Conversely, fulltime inspectors may not have been in practice for many years and thus may lack first-hand experience with newer technologies and the clinical relevance of testing.

CAP inspections are increasingly complex, detailed, and multifaceted because of the evolution of technology, the need to include regulatory requirements, and the effort to minimize inspector imprecision. The speed of inspection needs to be increased. The CAP checklist expands each year, along with expansion of the responsibility of laboratories. Although the Standards for Accreditation are relatively static, the checklists are frequently changed in response to input from the laboratory community. The checklists are not standards in and of themselves.

In laboratory conglomerates such as the VAMHCS, Web-based documentation allows for easier sharing among decentralized, geographically separated laboratories of the VAMHCS, and it also allows for centralized updates of the laboratory documentation. For example, because the procurement manual resides on the VAMHCS Intranet, there is no need to have paper copies on the wards for the inspectors to examine. The Intranet is easily accessible throughout the institutions. Any staff member who has access to the Intranet also has an immediate copy of the procurement manual. A backup copy of all the manuals is also available on the institutionwide VISTA computer system, if the Intranet is unavailable. The CAP does not require paper copies, recognizing that electronic versions have distinct advantages.

The VAMHCS Intranet serves a backup copy of the manual. Therefore, it is less likely that data will be lost. A secure Intranet firewall keeps out intruders. Intranet files can be upgraded to allow for multimedia presentations, including text search for key words, hyperlinks to other documents and PubMed references, and additional images, audio, and video. Use of the Intranet can speed up and improve the efficiency of the inspection process. The APS received no deficiencies during its last CAP inspection and was commended by the inspectors for the efficient organization of their documents.

\section{REFERENCES}

1. Rosai J, Ackerman LV. Ackerman's surgical pathology. 8th ed. St. Louis, MO: Mosby; 1996.

2. Moore GW, Berman JJ. Anatomic pathology data mining. In: Cios KJ, editor. Medical data mining and knowledge discovery. Heidelberg: Springer Verlag; 2000 (in press).

3. College of American Pathologists Web site. Available at: http://www.cap.org/. Accessed November 1, 2000.

4. Joint Commission for the Accreditation of Healthcare Organizations Web site. Available at: http://www.jcaho.org/. Accessed November 1, 2000.

5. College of American Pathologists. Standards for laboratory accreditation. Northfield, IL: College of American Pathologists; 1999.

6. College of American Pathologists. Quality improvement manual in anatomic pathology. Northfield, IL: College of American Pathologists; 1993.

7. Hoeltge GA, Dynek DA, Delahunty DC, McClatchey KD, Rabinovitch A, Robinowitz M, et al. Clinical laboratory technical procedure manuals. 3rd ed. Approved guideline GP2-A3. Vol 16, no 15. Wayne, PA: National Committee for Clinical Laboratory Standards; 1996.

8. The Johns Hopkins Autopsy Resource Web site. Available at: http://www.netautopsy.org. Accessed November 1, 2000. 
9. Hamlin WB. Requirements for accreditation by the College of American Pathologists Laboratory Accreditation Program. Arch Pathol Lab Med 1999;123:465-7.

10. Wagner LR. The College of American Pathologists, 19461996. The surveys program. Arch Pathol Lab Med 1997;121: 646-51.

11. Hamlin WB, Duckworth JK. The College of American Pathologists, 1946-1996: laboratory accreditation. Arch Pathol Lab Med 1997;121:745-53.

12. Wagner LR. The College of American Pathologists, 19461996. Interorganization relationships. Arch Pathol Lab Med 1997;121:1296-302.
13. Mark HF, Wells L. A laboratorian's view on the College of American Pathologists' Laboratory Accreditation Program for Clinical Laboratories. Ann Clin Lab Sci 1998;28:115-23.

14. Triol JH, Russell CD, Ashton PR. Health Care Financing Administration/American Society for Cytotechnology inspections: government assessment of cytology laboratory practice under the regulations of the Clinical Laboratory Improvement Amendments of 1988. Arch Pathol Lab Med 1997;121:264-6.

15. Department of Health and Human Services. Health Care Financing Administration. Clinical Laboratory Improvement Amendments of 1988. Final Rule, 57. Federal Register 7146 (1992) (codified at 42 CFR, 493). 